\title{
A general iterative algorithm for monotone operators with $\lambda$-hybrid mappings in Hilbert spaces
}

\section{Chung-Chien Hong*}

\section{"Correspondence:}

chong@mail.npust.edu.tw Department of Industrial

Management, National Pingtung

University of Science and

Technology, Pingtung, Taiwan

\begin{abstract}
Let $C$ be a nonempty closed convex subset of a Hilbert space $\mathcal{H}$, let $B, G$ be two set-valued maximal monotone operators on $C$ into $\mathcal{H}$, and let $g: \mathcal{H} \rightarrow \mathcal{H}$ be a $k$-contraction with $0<k<1$. $A: C \rightarrow \mathcal{H}$ is an $\alpha$-inverse strongly monotone mapping, $V: \mathcal{H} \rightarrow \mathcal{H}$ is a $\bar{\gamma}$-strongly monotone and L-Lipschitzian mapping with $\bar{\gamma}>0$ and $L>0, T: C \rightarrow C$ is a $\lambda$-hybrid mapping. In this paper, a general iterative scheme for approximating a point of $F(T) \cap(A+B)^{-1} 0 \cap G^{-1} 0 \neq \varnothing$ is introduced, where $F(T)$ is the set of fixed points of $T$, and a strong convergence theorem of the sequence generated by the iterative scheme is proved under suitable conditions. As applications of our strong convergence theorem, the related equilibrium and variational problems are also studied.
\end{abstract}

MSC: 47H05;47H10; 58E35

Keywords: fixed point; maximal monotone operator; inverse-strongly monotone mapping; resolvent; equilibrium problem; variational inequality

\section{Introduction}

Throughout this paper, $\mathcal{H}$ denotes a real Hilbert space, $C$ a nonempty closed convex subset of $\mathcal{H}, \mathbb{N}$ the set of all natural numbers and $\mathbb{R}$ the set of all real numbers. For a self-mapping $T$ on $\mathcal{H}, F(T)$ denotes the set of all fixed points of $T$.

A set-valued map $B: \mathcal{H} \rightarrow 2^{\mathcal{H}}$ with domain $\mathcal{D}(B):=\{x \in \mathcal{H}: B x \neq \varnothing\}$ is called monotone if

$$
\langle x-y, u-v\rangle \geq 0
$$

for all $x, y \in \mathcal{D}(B)$ and for any $u \in B x, v \in B y ; B$ is said to be maximal monotone if its graph $\{(x, u): x \in \mathcal{H}, u \in B(x)\}$ is not properly contained in the graph of any other monotone operator. For a positive real number $r$, the resolvent $J_{r}^{B}$ of a monotone operator $B$ for $r$ is a single-valued mapping $J_{r}^{B}: \mathcal{H} \rightarrow \mathcal{D}(B)$ defined by $z=J_{r}^{B}(x)$ if and only if $x \in z+r B z$, that is, $J_{r}^{B}(x)=(I+r B)^{-1}(x)$ for any $x \in \mathcal{H}$, where $I$ is the identity mapping on $\mathcal{H}$. The Yosida approximation $A_{r}$ of $B$ for $r>0$ is defined as $A_{r}=\frac{1}{r}\left(I-J_{r}^{B}\right)$. It is known [1] that $A_{r} x \in B\left(J_{r}^{B} x\right)$ for all $x \in \mathcal{H}$.

The fixed point theory for nonexpansive mappings can be applied to the problem of finding a zero point $v$ of a maximal monotone operator $B$ on $\mathcal{H}$, that is, finding a point

\section{Springer}

(02014 Hong; licensee Springer. This is an Open Access article distributed under the terms of the Creative Commons Attribution License (http://creativecommons.org/licenses/by/2.0), which permits unrestricted use, distribution, and reproduction in any medium, provided the original work is properly cited. 
$v \in \mathcal{H}$ satisfying $0 \in B(v)$. In the sequel, we shall denote the set of all zero points of $B$ by $B^{-1} 0$.

A self-mapping $V$ on $\mathcal{H}$ is called $\bar{\gamma}$-strongly monotone if there is a positive real number $\bar{\gamma}$ such that

$$
\langle x-y, V x-V y\rangle \geq \bar{\gamma}\|x-y\|^{2}, \quad \forall x, y \in \mathcal{H}
$$

$V$ is called $L$-Lipschitzian if there is a positive real number $L$ such that

$$
\|V x-V y\| \leq L\|x-y\|, \quad \forall x, y \in \mathcal{H} .
$$

A mapping $A: C \rightarrow \mathcal{H}$ is said to be $\alpha$-inverse strongly monotone if there is a positive real number $\alpha$ such that

$$
\langle x-y, A x-A y\rangle \geq \alpha\|A x-A y\|^{2}, \quad \forall x, y \in C .
$$

As easily seen, an $\alpha$-inverse strongly monotone mapping is $\frac{1}{\alpha}$-Lipschitzian on $C$.

For $\lambda \in \mathbb{R}$, a mapping $T: C \rightarrow \mathcal{H}$ is said to be $\lambda$-hybrid if

$$
\|T x-T y\|^{2} \leq\|x-y\|^{2}+\lambda\langle x-T x, y-T y\rangle, \quad \forall x, y \in C .
$$

When $\lambda=2, T$ is called nonspreading. It is known that $F(T)$ is closed and convex provided $T$ is a $\lambda$-hybrid self-mapping on $C, c f$. [2].

Recently, Lin and Takahashi [3] introduced an algorithm for finding a point $p_{0} \in(A+$ $B)^{-1} 0 \cap G^{-1} 0$, where $A$ is an $\alpha$-inverse strongly monotone mapping of $C$ into $\mathcal{H}$, and $B$, $G$ are two set-valued maximal monotone operators with $\mathcal{D}(B) \subset C$ and $\mathcal{D}(G) \subset C$. More precisely, let $g$ be a $k$-contraction and $V$ be a $\bar{\gamma}$-strongly monotone and $L$-Lipschitzian mapping. Choose $\mu, \gamma \in \mathbb{R}$ so that $0<\frac{2 \bar{\gamma}}{L^{2}}$ and $0<\gamma<\frac{\bar{\gamma}-\frac{L^{2} \mu}{2}}{k}$. Then the algorithm starts with any $x_{1} \in \mathcal{H}$ and generates a sequence $\left\{x_{n}\right\}$ iteratively by

$$
x_{n+1}=\alpha_{n} \gamma g\left(x_{n}\right)+\left(I-\alpha_{n} V\right) J_{\sigma_{n}}^{B}\left(I-\sigma_{n} A\right) J_{r_{n}}^{G} x_{n}, \quad n \in \mathbb{N}
$$

where $\left\{\alpha_{n}\right\} \subseteq(0,1),\left\{\sigma_{n}\right\} \subseteq(0, \infty)$, and $\left\{r_{n}\right\} \subseteq(0, \infty)$ satisfy

$$
\begin{aligned}
& \lim _{n \rightarrow \infty} \alpha_{n}=0, \quad \sum_{n=1}^{\infty} \alpha_{n}=\infty, \quad \sum_{n=1}^{\infty}\left|\alpha_{n}-\alpha_{n+1}\right|<\infty, \quad 0<a \leq \sigma_{n} \leq 2 \alpha, \\
& \sum_{n=1}^{\infty}\left|\sigma_{n}-\sigma_{n+1}\right|<\infty, \quad \liminf _{n \rightarrow \infty} r_{n}>0 \quad \text { and } \quad \sum_{n=1}^{\infty}\left|r_{n}-r_{n+1}\right|<\infty .
\end{aligned}
$$

They proved that $P_{(A+B)^{-1} 0 \cap G^{-1} 0}(I-V+\gamma g)$ has a unique fixed point $p_{0}$ in $\Omega$, and this $p_{0}$ is also a unique solution $p_{0} \in(A+B)^{-1} 0 \cap G^{-1} 0$ to the hierarchical variational inequality

$$
\langle(V-\gamma g) p, q-p\rangle, \quad \forall q \in \Omega
$$

As Lin and Takahashi said in [3], their idea for this algorithm comes from the works of Tian [4]. 
On the other hand, Manaka and Takahashi [5] used the algorithm

$$
x_{n+1}=\alpha_{n} x_{n}+\left(1-\alpha_{n}\right) T\left(J_{\sigma_{n}}^{B}\left(I-\sigma_{n} A\right) x_{n}\right)
$$

to find a point $p_{0} \in F(T) \cap(A+B)^{-1} 0$ for a nonspreading mapping $T$, an $\alpha$-inverse strongly monotone mapping $A$ and a maximal monotone operator $B$ under the conditions

$$
0<c \leq \alpha_{n} \leq d<1 \quad \text { and } \quad 0<a \leq \sigma_{n} \leq b<2 \alpha,
$$

where $a, b, c, d \in \mathbb{R}$ are fixed. They proved that the sequence $\left\{x_{n}\right\}$ constructed above converges weakly to a point $p \in F(T) \cap(A+B)^{-1} 0$.

Very recently, Liu et al. [6] modified the iterative scheme (2) to approximate a point $p \in F(T) \cap(A+B)^{-1} 0$ for a nonspreading mapping $T$, an $\alpha$-inverse strongly monotone mapping and a maximal monotone operator $B$. For any $u \in \mathcal{H}$, they put $x_{1}$ to be any point of $\mathcal{H}$ and define recursively for all $n \in \mathbb{N}$,

$$
\left\{\begin{array}{l}
z_{n}=J_{\alpha_{n}}^{B}\left(I-\sigma_{n} A\right) x_{n}, \\
y_{n}=\frac{1}{n} \sum_{i=0}^{n-1} T^{i} z_{n}, \\
x_{n+1}=\alpha_{n} u+\left(1-\alpha_{n}\right) y_{n},
\end{array}\right.
$$

where $\left\{\alpha_{n}\right\}$ is a suitable sequence in $[0,1]$.

Motivated by the above works, in this paper we introduce a general iterative scheme for approximating a point of $F(T) \cap(A+B)^{-1} 0 \cap G^{-1} 0 \neq \varnothing$, where $T$ is a $\lambda$-hybrid self-mapping on $C, A: C \rightarrow \mathcal{H}$ is an $\alpha$-inverse strongly monotone mapping and $B$ and $G$ are two maximal monotone operators. A strong convergence theorem of the sequence generated by our iterative scheme is proved under suitable conditions. Our result improves and generalizes the main theorem of Lin and Takahashi [3]. As applications of our strong convergence theorem, the related equilibrium and variational problems are also studied.

\section{Preliminaries}

In order to facilitate our investigation, in what follows we recall some basic facts. A mapping $T: C \rightarrow \mathcal{H}$ is said to be

(i) nonexpansive if

$$
\|T x-T y\| \leq\|x-y\|, \quad \forall x, y \in C ;
$$

(ii) firmly nonexpansive if

$$
\|T x-T y\|^{2} \leq\langle x-y, T x-T y\rangle, \quad \forall x, y \in C .
$$

The metric projection $P_{C}$ from $\mathcal{H}$ onto $C$ is the mapping that assigns each $x \in \mathcal{H}$ the unique point $P_{C} x$ in $C$ with the property

$$
\left\|x-P_{C} x\right\|=\min _{y \in C}\|y-x\| .
$$

It is known that $P_{C}$ is nonexpansive and characterized by the inequality: for any $x \in \mathcal{H}$,

$$
\left\langle x-P_{C} x, y-P_{C} x\right\rangle \leq 0, \quad \forall y \in C,
$$

cf. [7]. 
For any $x, y \in \mathcal{H}$, one has

$$
\|x+y\|^{2} \leq\|x\|^{2}+2\langle y, x+y\rangle
$$

Lemma 2.1 (Demiclosedness principle) [8] Let $T$ be a nonexpansive self-mapping on a nonempty closed convex subset $C$ of $\mathcal{H}$, and suppose that $\left\{x_{n}\right\}$ is a sequence in $C$ such that $\left\{x_{n}\right\}$ converges weakly to some $z \in C$ and $\lim _{n \rightarrow \infty}\left\|x_{n}-T x_{n}\right\|=0$. Then $T z=z$.

For $s>0$, the resolvent $J_{s}^{B}$ of the maximal monotone operator $B$ on $\mathcal{H}$ has the following properties, $c f .[9]$.

Lemma 2.2 Let $B$ be a maximal monotone operator on $\mathcal{H}$. Then, for any $s>0$,

(a) $J_{s}^{B}$ is single-valued and firmly nonexpansive;

(b) $\mathcal{D}\left(J_{s}^{B}\right)=\mathcal{H}$ and $F\left(J_{s}^{B}\right)=B^{-1} 0$.

The following lemma can be derived easily from the resolvent identity of a monotone operator $B$ :

$$
J_{s}^{B} x=J_{t}^{B}\left(\begin{array}{l}
t \\
-x \\
s
\end{array}+\left(1-\frac{t}{s}\right) J_{s}^{B} x\right) \quad \text { for any } s, t>0 \text { and any } x \in \mathcal{H}
$$

Lemma 2.3 Let $B$ be a monotone operator on $\mathcal{H}$. Then, for any $s, t \in \mathbb{R}$ with $s, t>0$ and for any $x \in \mathcal{H}$,

$$
\left\|J_{s}^{B} x-J_{t}^{B} x\right\| \leq \frac{|s-t|}{s}\left\|x-J_{s}^{B} x\right\|
$$

When $B$ is maximal monotone, a different proof may be found in Takahashi et al. [9].

Lemma 2.4 [5] Let $A: C \rightarrow \mathcal{H}$ be an $\alpha$-inverse strongly monotone mapping, and let $B$ be a maximal monotone operator on $\mathcal{H}$ with $\mathcal{D}(B) \subseteq C$. Then, for any $\sigma>0$, one has $(A+B)^{-1} 0=$ $F\left(J_{\sigma}^{B}(I-\sigma A)\right)$.

Lemma 2.5 [3] Let $A: C \rightarrow \mathcal{H}$ be an $\alpha$-inverse strongly monotone mapping. Then, for any $\sigma \in(0,2 \alpha],(I-\sigma A)$ is nonexpansive.

Lemma 2.6 [3] Let $g: \mathcal{H} \rightarrow \mathcal{H}$ be a $k$-contraction with $0<k<1$, let $V: \mathcal{H} \rightarrow \mathcal{H}$ be a $\bar{\gamma}$-strongly monotone and L-Lipschitzian mapping with $\bar{\gamma}>0$ and $L>0$, and let $\gamma$ be a real number satisfying $0<\gamma<\frac{\bar{\gamma}}{k}$. Then $V-\gamma g$ is a $(\bar{\gamma}-\gamma k)$-strongly monotone and $(L+\gamma k)$ Lipschitzian mapping. Furthermore, for any nonempty closed convex subset $\Omega$ of $\mathcal{H}, P_{\Omega}(I-$ $V+\gamma g$ ) has a unique fixed point $p_{0} \in \Omega$, which is also a unique solution of the variational inequality

$$
\langle(V-\gamma g) z, q-z\rangle \geq 0, \quad \forall q \in \Omega
$$

Lemma 2.7 [10] Let $\left\{s_{n}\right\}$ be a sequence of nonnegative real numbers satisfying

$$
s_{n+1} \leq\left(1-\alpha_{n}\right) s_{n}+\alpha_{n} \mu_{n}+v_{n}, \quad n \in \mathbb{N}
$$

where $\left\{\alpha_{n}\right\},\left\{\mu_{n}\right\}$ and $\left\{v_{n}\right\}$ verify the following conditions: 
(i) $\left\{\alpha_{n}\right\} \subseteq[0,1], \sum_{n=1}^{\infty} \alpha_{n}=\infty$;

(ii) $\lim \sup _{n \rightarrow \infty} \mu_{n} \leq 0$;

(iii) $\left\{v_{n}\right\} \subseteq[0, \infty)$ and $\sum_{n=1}^{\infty} v_{n}<\infty$.

Then $\lim _{n \rightarrow \infty} s_{n}=0$.

\section{Strong convergence theorems}

We begin the proof of the main result of this paper. As the proof is rather lengthy, we divide the proof into many assertions.

Theorem 3.1 Suppose that

(3.1.1) $G: C \rightarrow 2^{\mathcal{H}}$ and $B: C \rightarrow 2^{\mathcal{H}}$ are two maximal monotone operators with $\mathcal{D}(G) \subseteq C$ and $\mathcal{D}(B) \subseteq C$;

(3.1.2) $g: \mathcal{H} \rightarrow \mathcal{H}$ is a $k$-contraction, $A: C \rightarrow \mathcal{H}$ is an $\alpha$-inverse strongly monotone mapping, and $V: \mathcal{H} \rightarrow \mathcal{H}$ is a $\bar{\gamma}$-strongly monotone and L-Lipschitzian mapping with $\bar{\gamma}>0$ and $L>0$;

(3.1.3) $T: C \rightarrow C$ is a $\lambda$-hybrid mapping;

(3.1.4) $\Omega:=F(T) \cap(A+B)^{-1} 0 \cap G^{-1} 0 \neq \varnothing$;

(3.1.5) $\mu$ and $\gamma$ are two real numbers satisfying $0<\mu<\frac{2 \bar{\gamma}}{L^{2}}$ and $0<\gamma<\frac{\bar{\gamma}-\frac{L^{2} \mu}{2}}{k}$.

Start with any $x_{1} \in \mathcal{H}$ and define a sequence $\left\{x_{n}\right\}$ iteratively by

$$
\left\{\begin{array}{l}
z_{n}=J_{\sigma_{n}}^{B}\left(I-\sigma_{n} A\right) J_{r_{n}}^{G} x_{n}, \quad n \in \mathbb{N}, \\
y_{n}=\frac{1}{n} \sum_{i=0}^{n-1} T^{i} z_{n}, \quad n \in \mathbb{N}, \\
x_{n+1}=\alpha_{n} \gamma g\left(x_{n}\right)+\left(I-\alpha_{n} V\right) y_{n},
\end{array}\right.
$$

where the sequences $\left\{\alpha_{n}\right\},\left\{\sigma_{n}\right\}$ and $\left\{r_{n}\right\}$ verify the following conditions:

(3.1.6) $\left\{\alpha_{n}\right\}$ is a sequence in $[0,1]$ with $\lim _{n \rightarrow \infty} \alpha_{n}=0$ and $\sum_{n=1}^{\infty} \alpha_{n}=\infty$;

(3.1.7) $\left\{\sigma_{n}\right\}$ and $\left\{r_{n}\right\}$ are sequences in $(0, \infty)$ so that $\liminf _{n \rightarrow \infty} r_{n}>0$ and there are $a, b \in \mathbb{R}$ with $0<a \leq \sigma_{n} \leq b<2 \alpha$ for all $n \in \mathbb{N}$.

Then the sequence $\left\{x_{n}\right\}$ constructed by algorithm (6) converges strongly to a point $p_{0} \in \Omega$, where $p_{0}$ is the unique fixed point of $P_{\Omega}(I-V+\gamma g)$, and this point $p_{0}$ is also a unique solution of the hierarchical inequality

$$
\langle(V-\gamma g) p, q-p\rangle \geq 0, \quad \forall q \in \Omega
$$

Proof In what follows, $p$ is a point in $\Omega, \tau=\bar{\gamma}-\frac{L^{2} \mu}{2}$, and $u_{n}=J_{r_{n}}^{G} x_{n}$ for all $n \in \mathbb{N}$.

- Assertion (A): There is $N \in \mathbb{N}$ such that

$$
\left\|\left(I-\alpha_{n} V\right) y_{n}-\left(I-\alpha_{n} V\right) p\right\| \leq\left(1-\alpha_{n} \tau\right)\left\|y_{n}-p\right\| .
$$

Since $\lim _{n \rightarrow \infty} \alpha_{n}=0$, there is $N \in \mathbb{N}$ such that $1-\alpha_{n} \tau>0$ and $\mu-\alpha_{n}>0$ for all $n \geq N$. Then, as $V$ is $\bar{\gamma}$-strongly monotone and $L$-Lipschitzian, we have that for all $n \geq N$,

$$
\begin{aligned}
\| & \left(I-\alpha_{n} V\right) y_{n}-\left(I-\alpha_{n} V\right) p \|^{2} \\
& =\left\|\left(y_{n}-p\right)-\alpha_{n}\left(V y_{n}-V p\right)\right\|^{2} \\
& =\left\|y_{n}-p\right\|^{2}-2 \alpha_{n}\left\langle y_{n}-p, V y_{n}-V p\right\rangle+\alpha_{n}^{2}\left\|V y_{n}-V p\right\|^{2}
\end{aligned}
$$




$$
\begin{aligned}
& \leq\left\|y_{n}-p\right\|^{2}-2 \alpha_{n} \bar{\gamma}\left\|y_{n}-p\right\|^{2}+\alpha_{n}^{2} L^{2}\left\|y_{n}-p\right\|^{2} \\
& =\left(1-2 \alpha_{n} \tau-\alpha_{n} L^{2} \mu+\alpha_{n}^{2} L^{2}\right)\left\|y_{n}-p\right\|^{2} \\
& \leq\left(1-2 \alpha_{n} \tau+\alpha_{n}^{2} \tau^{2}\right)\left\|y_{n}-p\right\|^{2}-\alpha_{n} L^{2}\left(\mu-\alpha_{n}\right)\left\|y_{n}-p\right\|^{2} \\
& \leq\left(1-\alpha_{n} \tau\right)^{2}\left\|y_{n}-p\right\|^{2}
\end{aligned}
$$

and so the assertion holds.

- Assertion (B): The sequences $\left\{x_{n}\right\},\left\{y_{n}\right\},\left\{z_{n}\right\},\left\{u_{n}\right\},\left\{A u_{n}\right\},\left\{V y_{n}\right\},\left\{g\left(x_{n}\right)\right\}$ and $\left\{T^{n} z_{n}\right\}$ are bounded.

We firstly show that

$$
\left\|y_{n}-p\right\| \leq\left\|z_{n}-p\right\| \leq\left\|x_{n}-p\right\|, \quad \forall n \in \mathbb{N} .
$$

On account of $p=J_{\sigma_{n}}^{B}\left(I-\sigma_{n} A\right) p$ by Lemma 2.4, $p=J_{r_{n}}^{G} p$ by assumption and the facts that a resolvent is nonexpansive and $A$ is $\alpha$-inverse strongly monotone, we have

$$
\begin{aligned}
\left\|z_{n}-p\right\|^{2} & =\left\|J_{\sigma_{n}}^{B}\left(I-\sigma_{n} A\right) J_{r_{n}}^{G} x_{n}-J_{\sigma_{n}}^{B}\left(I-\sigma_{n} A\right) J_{r_{n}}^{G} p\right\|^{2} \\
& \leq\left\|\left(I-\sigma_{n} A\right) J_{r_{n}}^{G} x_{n}-\left(I-\sigma_{n} A\right) J_{r_{n}}^{G} p\right\|^{2} \\
& =\left\|J_{r_{n}}^{G} x_{n}-J_{r_{n}}^{G} p-\sigma_{n}\left(A J_{r_{n}}^{G} x_{n}-A J_{r_{n}}^{G} p\right)\right\|^{2} \\
& =\left\|J_{r_{n}}^{G} x_{n}-J_{r_{n}}^{G} p\right\|^{2}-2 \sigma_{n}\left\langle u_{n}-p, A u_{n}-A p\right\rangle+\sigma_{n}^{2}\left\|A u_{n}-A p\right\|^{2} \\
& \leq\left\|x_{n}-p\right\|^{2}-2 \sigma_{n} \alpha\left\|A u_{n}-A p\right\|^{2}+\sigma_{n}^{2}\left\|A u_{n}-A p\right\|^{2} \\
& =\left\|x_{n}-p\right\|^{2}-\sigma_{n}\left(2 \alpha-\sigma_{n}\right)\left\|A u_{n}-A p\right\|^{2} \\
& \leq\left\|x_{n}-p\right\|^{2},
\end{aligned}
$$

where the last inequality follows from the hypothesis that $2 \alpha \geq \sigma_{n}$. Therefore,

$$
\left\|z_{n}-p\right\| \leq\left\|x_{n}-p\right\|
$$

Since $T$ is $\lambda$-hybrid, we have, for any $n \in \mathbb{N}$,

$$
\left\|T z_{n}-p\right\|^{2}=\left\|T z_{n}-T p\right\|^{2} \leq\left\|z_{n}-p\right\|^{2}+\lambda\left\langle z_{n}-T z_{n}, p-T p\right\rangle=\left\|z_{n}-p\right\|^{2},
$$

and so, by induction, it comes easily that

$$
\left\|T^{i} z_{n}-p\right\| \leq\left\|z_{n}-p\right\| \quad \text { for all } i, n \in \mathbb{N} \text {. }
$$

Consequently,

$$
\begin{aligned}
\left\|y_{n}-p\right\| & =\left\|\frac{1}{n} \sum_{i=0}^{n-1} T^{i} z_{n}-p\right\| \\
& \leq \frac{1}{n} \sum_{i=0}^{n-1}\left\|T^{i} z_{n}-p\right\|
\end{aligned}
$$




$$
\begin{aligned}
& \leq \frac{1}{n} \sum_{i=0}^{n-1}\left\|z_{n}-p\right\| \\
& =\left\|z_{n}-p\right\| \leq\left\|x_{n}-p\right\| .
\end{aligned}
$$

Next, we show that $\left\{\left\|x_{n}-p\right\|\right\}$ is bounded. Indeed, as $p=\left(I-\alpha_{n} V\right) p+\alpha_{n} V p$, we have

$$
\begin{aligned}
& \left\|x_{n+1}-p\right\| \\
& \quad=\left\|\alpha_{n} \gamma g\left(x_{n}\right)+\left(I-\alpha_{n} V\right) y_{n}-\alpha_{n} V p-\left(I-\alpha_{n} V\right) p\right\| \\
& \quad \leq\left\|\alpha_{n}\left(\gamma g\left(x_{n}\right)-V p\right)\right\|+\left\|\left(I-\alpha_{n} V\right) y_{n}-\left(I-\alpha_{n} V\right) p\right\| \\
& \quad \leq \alpha_{n}\left\|\gamma g\left(x_{n}\right)-\gamma g(p)\right\|+\alpha_{n}\|\gamma g(p)-V p\|+\left\|\left(I-\alpha_{n} V\right) y_{n}-\left(I-\alpha_{n} V\right) p\right\| \\
& \quad \leq \alpha_{n} \gamma k\left\|x_{n}-p\right\|+\alpha_{n}\|\gamma g(p)-V p\|+\left\|\left(I-\alpha_{n} V\right) y_{n}-\left(I-\alpha_{n} V\right) p\right\|,
\end{aligned}
$$

which together with Assertion (A) implies that for all $n \geq N$,

$$
\begin{aligned}
\left\|x_{n+1}-p\right\| & \leq \alpha_{n} \gamma k\left\|x_{n}-p\right\|+\alpha_{n}\|\gamma g(p)-V p\|+\left(1-\alpha_{n} \tau\right)\left\|y_{n}-p\right\| \\
& \leq\left(1-\alpha_{n}(\tau-\gamma k)\right)\left\|x_{n}-p\right\|+\alpha_{n}\|\gamma g(p)-V p\| \\
& \leq\left\|x_{n}-p\right\|+\|\gamma g(p)-V p\|,
\end{aligned}
$$

from which we inductively deduce that

$$
\left\|x_{n}-p\right\| \leq\left\|x_{1}-p\right\|+\|\gamma g(p)-V p\|
$$

Thus, $\left\{x_{n}\right\}$ is bounded, and so are $\left\{y_{n}\right\},\left\{z_{n}\right\}$ by (9). And then the boundedness of $\left\{T^{n} z_{n}\right\}$ follows from (12). The fact that $\left\{V y_{n}\right\}$ and $\left\{g\left(x_{n}\right)\right\}$ are bounded is due to the fact that $V$ and $g$ are $L$-Lipschitzian and $k$-contraction, respectively.

Finally, from $\left\|u_{n}-p\right\|=\left\|J_{\sigma_{n}}^{G} x_{n}-J_{\sigma_{n}}^{G} p\right\| \leq\left\|x_{n}-p\right\|$, we deduce that $\left\{u_{n}\right\}$ is bounded, and $\left\{A u_{n}\right\}$ is bounded comes from $A$ is $\frac{1}{\alpha}$-Lipschitzian.

- Assertion (C): $\lim _{n \rightarrow \infty}\left\|x_{n+1}-x_{n}\right\|=0$.

We at first show that $\lim _{n \rightarrow \infty}\left\|y_{n+1}-y_{n}\right\|=0$. By Assertion (B), we can choose a positive real number $M$ so that

$$
\max \left\{\sup _{n \in \mathbb{N}}\left\|T^{n} z_{n}\right\|, \sup _{n \in \mathbb{N}}\left\|y_{n}\right\|\right\} \leq M .
$$

Then, for all $n \in \mathbb{N}$,

$$
\begin{aligned}
\left\|y_{n+1}-y_{n}\right\| & =\left\|\frac{1}{n+1} \sum_{i=0}^{n} T^{i} z_{n}-\frac{1}{n} \sum_{i=0}^{n-1} T^{i} z_{n}\right\| \\
& \leq \frac{1}{n+1}\left\|T^{n} z_{n}\right\|+\left(\frac{1}{n}-\frac{1}{n+1}\right)\left\|\sum_{i=0}^{n-1} T^{i} z_{n}\right\| \\
& =\frac{1}{n+1}\left\|T^{n} z_{n}\right\|+\frac{1}{n+1}\left\|y_{n}\right\| \leq \frac{2 M}{n+1} .
\end{aligned}
$$

Hence $\lim _{n \rightarrow \infty}\left\|y_{n+1}-y_{n}\right\|=0$. 
Now, for all $n \geq N$, we have from Assertion (A) that

$$
\begin{aligned}
& \left\|x_{n+2}-x_{n+1}\right\| \\
& =\left\|\alpha_{n+1} \gamma g\left(x_{n+1}\right)+\left(I-\alpha_{n+1} V\right) y_{n+1}-\alpha_{n} \gamma g\left(x_{n}\right)-\left(I-\alpha_{n} V\right) y_{n}\right\| \\
& \leq\left\|\alpha_{n+1} \gamma g\left(x_{n+1}\right)-\alpha_{n+1} \gamma g\left(x_{n}\right)\right\|+\left\|\alpha_{n+1} \gamma g\left(x_{n}\right)-\alpha_{n} \gamma g\left(x_{n}\right)\right\| \\
& \quad+\left\|\left(I-\alpha_{n+1} V\right) y_{n+1}-\left(I-\alpha_{n+1} V\right) y_{n}\right\|+\left\|\left(I-\alpha_{n+1} V\right) y_{n}-\left(I-\alpha_{n} V\right) y_{n}\right\| \\
& \leq \alpha_{n+1} \gamma k\left\|x_{n+1}-x_{n}\right\|+\gamma\left|\alpha_{n+1}-\alpha_{n}\right|\left\|g\left(x_{n}\right)\right\|+\left(1-\alpha_{n+1} \tau\right)\left\|y_{n+1}-y_{n}\right\| \\
& \quad+\left|\alpha_{n+1}-\alpha_{n}\right|\left\|V y_{n}\right\| .
\end{aligned}
$$

Consequently, using condition (3.1.6), Assertion (B) and $\lim _{n \rightarrow \infty}\left\|y_{n+1}-y_{n}\right\|=0$, we get $\lim _{n \rightarrow \infty}\left\|x_{n+1}-x_{n}\right\|=0$.

- Assertion (D): $\lim _{n \rightarrow \infty}\left\|A u_{n}-A p\right\|=0$.

Using (5), it follows from (8), (9) and (11) that

$$
\begin{aligned}
&\left\|x_{n+1}-p\right\|^{2} \\
&=\left\|\left(I-\alpha_{n} V\right) y_{n}-\left(I-\alpha_{n} V\right) p+\alpha_{n} \gamma g\left(x_{n}\right)-V p\right\|^{2} \\
& \leq\left\|\left(I-\alpha_{n} V\right) y_{n}-\left(I-\alpha_{n} V\right) p\right\|^{2}+2 \alpha_{n}\left\langle\gamma g\left(x_{n}\right)-V p, x_{n+1}-p\right\rangle \quad \text { by }(5) \\
& \leq\left(1-\alpha_{n} \tau\right)^{2}\left\|y_{n}-p\right\|^{2}+2 \alpha_{n}\left\langle\gamma g\left(x_{n}\right)-V p, x_{n+1}-p\right\rangle \quad \text { by }(8) \\
& \leq\left(1-\alpha_{n} \tau\right)^{2}\left\|z_{n}-p\right\|^{2}+2 \alpha_{n}\left\langle\gamma g\left(x_{n}\right)-V p, x_{n+1}-p\right\rangle \quad \text { by }(9) \\
& \leq\left(1-\alpha_{n} \tau\right)^{2}\left(\left\|x_{n}-p\right\|^{2}-\sigma_{n}\left(2 \alpha-\sigma_{n}\right)\left\|A u_{n}-A p\right\|^{2}\right) \\
&+2 \alpha_{n}\left\langle\gamma g\left(x_{n}\right)-V p, x_{n+1}-p\right\rangle \quad \text { by }(11) \\
& \leq\left(1-\alpha_{n} \tau\right)^{2}\left\|x_{n}-p\right\|^{2}-\left(1-\alpha_{n} \tau\right)^{2} \sigma_{n}\left(2 \alpha-\sigma_{n}\right)\left\|A u_{n}-A p\right\|^{2} \\
&+2 \alpha_{n}\left\langle\gamma g\left(x_{n}\right)-V p, x_{n+1}-p\right\rangle \\
& \leq\left\|x_{n}-p\right\|^{2}+\alpha_{n}^{2} \tau^{2}\left\|x_{n}-p\right\|^{2}-\left(1-\alpha_{n} \tau\right)^{2} \sigma_{n}\left(2 \alpha-\sigma_{n}\right)\left\|A u_{n}-A p\right\|^{2} \\
&+2 \alpha_{n}\left\langle\gamma g\left(x_{n}\right)-V p, x_{n+1}-p\right\rangle .
\end{aligned}
$$

Hence, on account of $0<a \leq \sigma_{n} \leq b<2 \alpha$ for all $n \in \mathbb{N}$, we have

$$
\begin{aligned}
(1- & \left.\alpha_{n} \tau\right)^{2} a(2 \alpha-b)\left\|A u_{n}-A p\right\|^{2} \\
\leq & \left(1-\alpha_{n} \tau\right)^{2} \sigma_{n}\left(2 \alpha-\sigma_{n}\right)\left\|A u_{n}-A p\right\|^{2} \\
\leq & \left\|x_{n}-p\right\|^{2}-\left\|x_{n+1}-p\right\|^{2}+\alpha_{n}^{2} \tau^{2}\left\|x_{n}-p\right\|^{2} \\
& +2 \alpha_{n}\left\langle\gamma g\left(x_{n}\right)-V p, x_{n+1}-p\right\rangle \\
\leq & \left\|x_{n}-x_{n+1}\right\|\left(\left\|x_{n}-p\right\|+\left\|x_{n+1}-p\right\|\right)+\alpha_{n}^{2} \tau^{2}\left\|x_{n}-p\right\|^{2} \\
& +2 \alpha_{n}\left\langle\gamma g\left(x_{n}\right)-V p, x_{n+1}-p\right\rangle,
\end{aligned}
$$

which together with Assertions (B) and (C) implies that $\lim _{n \rightarrow \infty}\left\|A u_{n}-A p\right\|=0$.

- Assertion (E): $\lim _{n \rightarrow \infty}\left\|x_{n}-y_{n}\right\|=\lim _{n \rightarrow \infty}\left\|x_{n}-u_{n}\right\|=0$. 
From

$$
\begin{aligned}
\left\|x_{n}-y_{n}\right\| & \leq\left\|x_{n}-x_{n+1}\right\|+\left\|x_{n+1}-y_{n}\right\| \\
& =\left\|x_{n}-x_{n+1}\right\|+\left\|\alpha_{n} \gamma g\left(x_{n}\right)+\left(I-\alpha_{n} V\right) y_{n}-y_{n}\right\| \\
& =\left\|x_{n}-x_{n+1}\right\|+\alpha_{n}\left\|\gamma g\left(x_{n}\right)-V y_{n}\right\|,
\end{aligned}
$$

condition (3.1.6) and Assertions (B) and (C), we conclude that $\lim _{n \rightarrow \infty}\left\|x_{n}-y_{n}\right\|=0$. As $J_{r_{n}}^{G}$ is firmly nonexpansive, one has

$$
\begin{aligned}
2\left\|u_{n}-p\right\|^{2} & =2\left\|J_{r_{n}}^{G} x_{n}-J_{r_{n}}^{G} p\right\|^{2} \\
& \leq 2\left\langle x_{n}-p, u_{n}-p\right\rangle \\
& =\left\|x_{n}-p\right\|^{2}+\left\|u_{n}-p\right\|^{2}-\left\|u_{n}-x_{n}\right\|^{2},
\end{aligned}
$$

and so

$$
\left\|u_{n}-p\right\|^{2} \leq\left\|x_{n}-p\right\|^{2}-\left\|u_{n}-x_{n}\right\|^{2}
$$

In addition, since $A$ is $\alpha$-inverse strongly monotone, we see from (10) that

$$
\begin{aligned}
\left\|z_{n}-p\right\|^{2} & \leq\left\|J_{r_{n}}^{G} x_{n}-J_{r_{n}}^{G} p\right\|^{2}-2 \sigma_{n}\left\langle u_{n}-p, A u_{n}-A p\right\rangle+\sigma_{n}^{2}\left\|A u_{n}-A p\right\|^{2} \\
& =\left\|u_{n}-p\right\|^{2}-2 \sigma_{n}\left\langle u_{n}-p, A u_{n}-A p\right\rangle+\sigma_{n}^{2}\left\|A u_{n}-A p\right\|^{2} \\
& \leq\left\|u_{n}-p\right\|^{2}-2 \sigma_{n} \alpha\left\|A u_{n}-A p\right\|^{2}+\sigma_{n}^{2}\left\|A u_{n}-A p\right\|^{2} \\
& =\left\|u_{n}-p\right\|^{2}-\sigma_{n}\left(2 \alpha-\sigma_{n}\right)\left\|A u_{n}-A p\right\|^{2} .
\end{aligned}
$$

Then (13), (14) and (15) give us that

$$
\begin{aligned}
\left\|x_{n+1}-p\right\|^{2} & \\
\leq & \left(1-\alpha_{n} \tau\right)^{2}\left\|z_{n}-p\right\|^{2}+2 \alpha_{n}\left\langle\gamma g\left(x_{n}\right)-V p, x_{n+1}-p\right\rangle \quad \text { by }(13) \\
\leq & \left(1-\alpha_{n} \tau\right)^{2}\left(\left\|u_{n}-p\right\|^{2}-\sigma_{n}\left(2 \alpha-\sigma_{n}\right)\left\|A u_{n}-A p\right\|^{2}\right) \quad \text { by }(15) \\
& +2 \alpha_{n}\left\langle\gamma g\left(x_{n}\right)-V p, x_{n+1}-p\right\rangle \\
\leq & \left(1-\alpha_{n} \tau\right)^{2}\left(\left\|x_{n}-p\right\|^{2}-\left\|u_{n}-x_{n}\right\|^{2}\right)-\left(1-\alpha_{n} \tau\right)^{2} \sigma_{n}\left(2 \alpha-\sigma_{n}\right)\left\|A u_{n}-A p\right\|^{2} \\
& +2 \alpha_{n}\left\langle\gamma g\left(x_{n}\right)-V p, x_{n+1}-p\right\rangle \quad \text { by }(14) \\
\leq & \left\|x_{n}-p\right\|^{2}+\alpha_{n}^{2} \tau^{2}\left\|x_{n}-p\right\|^{2}-\left(1-\alpha_{n} \tau\right)^{2}\left\|u_{n}-x_{n}\right\|^{2} \\
& -\left(1-\alpha_{n} \tau\right)^{2} \sigma_{n}\left(2 \alpha-\sigma_{n}\right)\left\|A u_{n}-A p\right\|^{2}+2 \alpha_{n}\left\langle\gamma g\left(x_{n}\right)-V p, x_{n+1}-p\right\rangle .
\end{aligned}
$$

Therefore

$$
\begin{aligned}
& \left(1-\alpha_{n} \tau\right)^{2}\left\|u_{n}-x_{n}\right\|^{2} \\
& \quad \leq\left\|x_{n}-p\right\|^{2}-\left\|x_{n+1}-p\right\|^{2}+\alpha_{n}^{2} \tau^{2}\left\|x_{n}-p\right\|^{2} \\
& \quad-\left(1-\alpha_{n} \tau\right)^{2} \sigma_{n}\left(2 \alpha-\sigma_{n}\right)\left\|A u_{n}-A p\right\|^{2}+2 \alpha_{n}\left\langle\gamma g\left(x_{n}\right)-V p, x_{n+1}-p\right\rangle
\end{aligned}
$$




$$
\begin{aligned}
\leq & \left\|x_{n}-x_{n+1}\right\|\left(\left\|x_{n}-p\right\|+\left\|x_{n+1}-p\right\|\right)+\alpha_{n}^{2} \tau^{2}\left\|x_{n}-p\right\|^{2} \\
& -\left(1-\alpha_{n} \tau\right)^{2} \sigma_{n}\left(2 \alpha-\sigma_{n}\right)\left\|A u_{n}-A p\right\|^{2}+2 \alpha_{n}\left\langle\gamma g\left(x_{n}\right)-V p, x_{n+1}-p\right\rangle .
\end{aligned}
$$

By Assertions (C), (D) and condition (3.1.6), we obtain

$$
\lim _{n \rightarrow \infty}\left\|x_{n}-u_{n}\right\|=0
$$

- Assertion (F): $\Omega$ is a nonempty closed convex subset of $\mathcal{H}$.

Since $\Omega$ is nonempty by assumption, it suffices to show that $\Omega$ is closed and convex. From Lemma 2.4, we have that for any $\sigma>0$,

$$
(A+B)^{-1} 0=F\left(J_{\sigma}^{B}(I-\sigma A)\right) .
$$

In case $0<\sigma \leq 2 \alpha$, we have that $I-\sigma A$ is nonexpansive by Lemma 2.5. Then $J_{\sigma}^{B}(I-\sigma A)$ is nonexpansive, and so $(A+B)^{-1} 0=F\left(J_{\sigma}^{B}(I-\sigma A)\right)$ is closed and convex. In the like manner, for any $r>0, G^{-1} 0=F\left(J_{r}^{G}\right)$ is closed and convex. Besides, it is shown in [2] that $F(T)$ is closed and convex. Hence comes the conclusion for $\Omega$.

- Assertion (G): $P_{\Omega}(I-V+\gamma g)$ has a unique fixed point $p_{0}$ in $\Omega$, and this $p_{0}$ is also a unique solution $p_{0} \in \Omega$ to the hierarchical variational inequality (7)

$$
\langle(V-\gamma g) p, q-p\rangle \geq 0, \quad \forall q \in \Omega
$$

Taking into account that $\Omega$ is a nonempty closed convex subset, the conclusion follows from Lemma 2.6.

- Assertion (H): Let $p_{0}$ be the unique solution to the hierarchical variational inequality (7). Then

$$
\limsup _{n \rightarrow \infty}\left\langle(V-\gamma g) p_{0}, x_{n}-p_{0}\right\rangle \leq 0
$$

Choose a subsequence $\left\{x_{n_{i}}\right\}$ of $\left\{x_{n}\right\}$ so that

$$
\limsup _{n \rightarrow \infty}\left\langle(V-\gamma g) p_{0}, x_{n}-p_{0}\right\rangle=\lim _{i \rightarrow \infty}\left\langle(V-\gamma g) p_{0}, x_{n_{i}}-p_{0}\right\rangle
$$

and $\left\{x_{n_{i}}\right\}$ converges weakly to $w \in \mathcal{H}$. In view of Assertion (E), we see that both of the sequences $\left\{u_{n_{i}}\right\}$ and $\left\{y_{n_{i}}\right\}$ converge weakly to $w$. We at first show that $w \in F(T)$. Since $T$ is $\lambda$-hybrid, one has $\|T x-T y\|^{2} \leq\|x-y\|^{2}+\lambda\langle x-T x, y-T y\rangle$ for all $x, y \in C$. Express $\lambda$ as $\lambda=2(1-\delta)$. Then, for all $x, y \in C$, we have

$$
\begin{aligned}
& \|T x-T y\|^{2} \\
& \quad \leq\|x-T y+T y-y\|^{2}+2(1-\delta)\langle x-T x, y-T y\rangle \\
& \quad=\|x-T y\|^{2}+2\langle x-T y, T y-y\rangle+\|T y-y\|^{2}+2(1-\delta)\langle x-T x, y-T y\rangle \\
& \quad=\|x-T y\|^{2}+\|T y-y\|^{2}+2\langle x-(1-\delta) x+(1-\delta) T x-T y, T y-y\rangle \\
& \quad=\|x-T y\|^{2}+\|T y-y\|^{2}+2\langle\delta x+(1-\delta) T x-T y, T y-y\rangle
\end{aligned}
$$


that is,

$$
0 \leq\|x-T y\|^{2}-\|T x-T y\|^{2}+\|T y-y\|^{2}+2\langle\delta x+(1-\delta) T x-T y, T y-y\rangle .
$$

In particular, for all $i \in \mathbb{N}$, all $z_{n}$ and all $y \in C$,

$$
\begin{aligned}
0 \leq & \left\|T^{i} z_{n}-T y\right\|^{2}-\left\|T^{i+1} z_{n}-T y\right\|^{2}+\|T y-y\|^{2} \\
& +2\left\langle\delta T^{i} z_{n}+(1-\delta) T^{i+1} z_{n}-T y, T y-y\right\rangle .
\end{aligned}
$$

Summing these inequalities from $i=0$ to $n-1$ and dividing by $n$, we obtain

$$
\begin{aligned}
0 \leq & \frac{1}{n}\left(\left\|z_{n}-T y\right\|^{2}-\left\|T^{n} z_{n}-T y\right\|^{2}\right)+\|T y-y\|^{2} \\
& +2\left\langle\delta y_{n}+(1-\delta)\left(\frac{n+1}{n} y_{n+1}-\frac{z_{n}}{n}\right)-T y, T y-y\right\rangle .
\end{aligned}
$$

Replacing $n$ with $n_{i}$ in (16) and letting $i \rightarrow \infty$, we get via Assertion (B) that

$$
0 \leq\|T y-y\|^{2}+2\langle\delta w+(1-\delta) w-T y, T y-y\rangle .
$$

Putting $y=w$ in (17), we arrive at $0 \leq-\|T w-w\|^{2}$. This shows that $w \in F(T)$.

Since $0<a \leq \sigma_{n_{i}} \leq b<2 \alpha,\left\{\sigma_{n_{i}}\right\}$ has a convergent subsequence. For simplicity, we assume that $\left\{\sigma_{n_{i}}\right\}$ converges to a number $\sigma \in[a, b]$. Note that for all $n \in \mathbb{N}$,

$$
\begin{aligned}
& \left\|J_{\sigma}^{B}(I-\sigma A) u_{n}-z_{n}\right\| \\
& \quad \leq\left\|J_{\sigma}^{B}(I-\sigma A) u_{n}-J_{\sigma}^{B}\left(I-\sigma_{n} A\right) u_{n}\right\|+\left\|J_{\sigma}^{B}\left(I-\sigma_{n} A\right) u_{n}-z_{n}\right\| \\
& \quad \leq\left\|(I-\sigma A) u_{n}-\left(I-\sigma_{n} A\right) u_{n}\right\|+\left\|J_{\sigma}^{B}\left(I-\sigma_{n} A\right) u_{n}-J_{\sigma_{n}}^{B}\left(I-\sigma_{n} A\right) u_{n}\right\| \\
& \quad \leq\left|\sigma_{n}-\sigma\right|\left\|A u_{n}\right\|+\frac{\left|\sigma_{n}-\sigma\right|}{\sigma}\left\|J_{\sigma}^{B} v_{n}-v_{n}\right\|,
\end{aligned}
$$

where $v_{n}=\left(I-\sigma_{n} A\right) u_{n}$. Furthermore, we have for all $n \in \mathbb{N}$,

$$
\begin{aligned}
\left\|J_{\sigma}^{B} v_{n}-v_{n}\right\| & \leq\left\|J_{\sigma}^{B} v_{n}-p\right\|+\left\|v_{n}-p\right\| \\
& =\left\|J_{\sigma}^{B} v_{n}-J_{\sigma}^{B} p\right\|+\left\|v_{n}-p\right\| \\
& \leq 2\left\|v_{n}-p\right\|
\end{aligned}
$$

and

$$
\begin{aligned}
\left\|v_{n}-p\right\| & =\left\|\left(I-\sigma_{n} A\right) u_{n}-\left(I-\sigma_{n} A\right) p-\sigma_{n} A p\right\| \\
& \leq\left\|u_{n}-p\right\|+b\|A p\| \quad \text { by Lemma } 2.5 .
\end{aligned}
$$

Hence, $\left\{J_{\sigma}^{B} v_{n}-v_{n}\right\}$ is bounded. Now, replace $n$ with $n_{i}$ in (18) and note that both $\left\{A u_{n_{i}}\right\}$ and $\left\{U_{\sigma}^{B} v_{n_{i}}-v_{n_{i}}\right\}$ are bounded. Letting $i \rightarrow \infty$, we get that

$$
\left\|J_{\sigma}^{B}(I-\sigma A) u_{n_{i}}-u_{n_{i}}\right\| \rightarrow 0
$$


Applying Lemma 2.1 to the nonexpansive mapping $J_{\sigma}^{B}(I-\sigma A)$, we conclude that $w=J_{\sigma}^{B}(I-$ $\sigma A) w$, that is, $w \in(A+B)^{-1} 0$.

We now show that $w \in G^{-1} 0$. Since $G$ is a maximal monotone operator, the Yosida approximation $A_{r} x=\frac{x-J_{r}^{G} x}{r}$ of $G$ for $r>0$ is in $F\left(J_{r}^{G}(x)\right)$. So, for any $(z, v) \in G$, one has

$$
\left\langle z-u_{n_{i}}, v-\frac{x_{n_{i}}-u_{n_{i}}}{r_{n_{i}}}\right\rangle \geq 0
$$

Since $\liminf _{n \rightarrow \infty} r_{n}>0,\left\{u_{n_{i}}\right\}$ converges weakly to $w$ and $x_{n_{i}}-u_{n_{i}} \rightarrow 0$, we have

$$
\langle z-w, v\rangle \geq 0,
$$

and then the maximality of $T$ shows that $0 \in G w$, that is, $0 \in G^{-1} 0$.

In summary, we have shown that $w \in \Omega$, and so, by Assertion (G), we conclude that

$$
\begin{aligned}
\limsup _{n \rightarrow \infty}\left\langle(V-\gamma g) p_{0}, x_{n}-p_{0}\right\rangle & =\lim _{i \rightarrow \infty}\left\langle(V-\gamma g) p_{0}, x_{n_{i}}-p_{0}\right\rangle \\
& =\left\langle(V-\gamma g) p_{0}, w-p_{0}\right\rangle \geq 0 .
\end{aligned}
$$

- Assertion (I): The sequence $\left\{x_{n}\right\}$ converges strongly to $p_{0}$.

Replacing $p$ with $p_{0}$ in (13), we have

$$
\begin{aligned}
\left\|x_{n+1}-p_{0}\right\|^{2} \leq & \left(1-\alpha_{n} \tau\right)^{2}\left\|z_{n}-p_{0}\right\|^{2}+2 \alpha_{n}\left\langle\gamma g\left(x_{n}\right)-V p_{0}, x_{n+1}-p_{0}\right\rangle \\
\leq & \left(1-\alpha_{n} \tau\right)^{2}\left\|z_{n}-p_{0}\right\|^{2}+2 \alpha_{n} \gamma k\left\|x_{n}-p_{0}\right\|\left\|x_{n+1}-p_{0}\right\| \\
& +2 \alpha_{n}\left\langle\gamma g\left(p_{0}\right)-V p_{0}, x_{n+1}-p_{0}\right\rangle \\
\leq & \left(1-\alpha_{n} \tau\right)^{2}\left\|x_{n}-p_{0}\right\|^{2}+\alpha_{n} \gamma k\left(\left\|x_{n}-p_{0}\right\|^{2}+\left\|x_{n+1}-p_{0}\right\|^{2}\right) \\
& +2 \alpha_{n}\left\langle\gamma g\left(p_{0}\right)-V p_{0}, x_{n+1}-p_{0}\right\rangle \text { by }(9) \\
= & \left(\left(1-\alpha_{n} \tau\right)^{2}+\alpha_{n} \gamma k\right)\left\|x_{n}-p_{0}\right\|^{2}+\alpha_{n} \gamma k\left\|x_{n+1}-p_{0}\right\|^{2} \\
& +2 \alpha_{n}\left\langle\gamma g\left(p_{0}\right)-V p_{0}, x_{n+1}-p_{0}\right\rangle .
\end{aligned}
$$

Hence

$$
\begin{aligned}
\left\|x_{n+1}-p_{0}\right\|^{2} \leq & \frac{1-2 \alpha_{n} \tau+\left(\alpha_{n} \tau\right)^{2}+\alpha_{n} \gamma k}{1-\alpha_{n} \gamma k}\left\|x_{n}-p_{0}\right\|^{2} \\
& +\frac{2 \alpha_{n}}{1-\alpha_{n} \gamma k}\left\langle\gamma g\left(p_{0}\right)-V p_{0}, x_{n+1}-p_{0}\right\rangle \\
= & \left(1-\frac{2(\tau-\gamma k) \alpha_{n}}{1-\alpha_{n} \gamma k}\right)\left\|x_{n}-p_{0}\right\|^{2}+\frac{\left(\alpha_{n} \tau\right)^{2}}{1-\alpha_{n} \gamma k}\left\|x_{n}-p_{0}\right\|^{2} \\
& +\frac{2 \alpha_{n}}{1-\alpha_{n} \gamma k}\left\langle\gamma g\left(p_{0}\right)-V p_{0}, x_{n+1}-p_{0}\right\rangle \\
= & \left(1-\beta_{n}\right)\left\|x_{n}-p_{0}\right\|^{2} \\
& +\beta_{n}\left(\frac{\alpha_{n} \tau^{2}\left\|x_{n}-p_{0}\right\|^{2}}{2(\tau-\gamma k)}+\frac{1}{\tau-\gamma k}\left\langle\gamma g\left(p_{0}\right)-V p_{0}, x_{n+1}-p_{0}\right\rangle\right),
\end{aligned}
$$


where $\beta_{n}=\frac{2(\tau-\gamma k) \alpha_{n}}{1-\alpha_{n} \gamma k}$. Since $\sum_{n=1}^{\infty} \beta_{n}=\infty$, and since

$$
\limsup _{n \rightarrow \infty}\left(\frac{\alpha_{n} \tau^{2}\left\|x_{n}-p_{0}\right\|^{2}}{2(\tau-\gamma k)}+\frac{1}{\tau-\gamma k}\left\langle\gamma g\left(p_{0}\right)-V p_{0}, x_{n+1}-p_{0}\right\rangle\right) \leq 0,
$$

by Assertion $(\mathrm{H})$, it follows from Lemma 2.7 that $\left\{x_{n}\right\}$ converges strongly to $p_{0}$. This completes the proof.

When $T$ is the identity mapping, the theorem reduces to the following corollary.

\section{Corollary 3.2 Suppose that}

(3.2.1) $G: C \rightarrow 2^{\mathcal{H}}$ and $B: C \rightarrow 2^{\mathcal{H}}$ are two maximal monotone operators with $\mathcal{D}(G) \subseteq C$ and $\mathcal{D}(B) \subseteq C$;

(3.2.2) $g: \mathcal{H} \rightarrow \mathcal{H}$ is a $k$-contraction, $A: C \rightarrow \mathcal{H}$ is an $\alpha$-inverse strongly monotone mapping, and $V: \mathcal{H} \rightarrow \mathcal{H}$ is a $\bar{\gamma}$-strongly monotone and L-Lipschitzian mapping with $\bar{\gamma}>0$ and $L>0$;

(3.2.3) $\Omega:=(A+B)^{-1} 0 \cap G^{-1} 0 \neq \varnothing$;

(3.2.4) $\mu$ and $\gamma$ are two real numbers satisfying $0<\mu<\frac{2 \bar{\gamma}}{L^{2}}$ and $0<\gamma<\frac{\bar{\gamma}-\frac{L^{2} \mu}{2}}{k}$. Start with any $x_{1} \in \mathcal{H}$ and define a sequence $\left\{x_{n}\right\}$ iteratively by

$$
\left\{\begin{array}{l}
y_{n}=J_{\sigma_{n}}^{B}\left(I-\sigma_{n} A\right) J_{r_{n}}^{G} x_{n}, \quad n \in \mathbb{N}, \\
x_{n+1}=\alpha_{n} \gamma g\left(x_{n}\right)+\left(I-\alpha_{n} V\right) y_{n},
\end{array}\right.
$$

where the sequences $\left\{\alpha_{n}\right\},\left\{\sigma_{n}\right\}$ and $\left\{r_{n}\right\}$ verify the following conditions:

(3.2.5) $\left\{\alpha_{n}\right\}$ is a sequence in $[0,1]$ with $\lim _{n \rightarrow \infty} \alpha_{n}=0$ and $\sum_{n=1}^{\infty} \alpha_{n}=\infty$;

(3.2.6) $\left\{\sigma_{n}\right\}$ and $\left\{r_{n}\right\}$ are sequences in $(0, \infty)$ so that $\liminf _{n \rightarrow \infty} r_{n}>0$ and there are $a, b \in \mathbb{R}$ with $0<a \leq \sigma_{n} \leq b<2 \alpha$ for all $n \in \mathbb{N}$.

Then $P_{\Omega}(I-V+\gamma g)$ has a unique fixed point $p_{0}$ in $\Omega$, and this $p_{0}$ is also a unique solution $p_{0} \in \Omega$ to the hierarchical variational inequality

$$
\langle(V-\gamma g) p, q-p\rangle, \quad \forall q \in \Omega
$$

Here we would like to remark that Corollary 3.2 is related to Theorem 8 in Lin and Takahashi [3], although our conditions (3.2.5) and (3.2.6) are different from the corresponding ones in [3].

\section{Applications}

In this section, we shall apply Theorem 3.1 to study the related equilibrium problem. Let $f: C \times C \rightarrow \mathbb{R}$ and $A: C \rightarrow \mathcal{H}$. Then a generalized equilibrium problem is the problem of finding $\hat{x} \in C$ such that

$$
f(\hat{x}, y)+\langle A \hat{x}, y-\hat{x}\rangle \geq 0, \quad \forall y \in C
$$

The solution set for Eq. (19) is denoted by $\operatorname{EP}(f, A)$, that is,

$$
\mathrm{EP}(f, A)=\{\hat{x} \in C: f(\hat{x}, y)+\langle A \hat{x}, y-\hat{x}\rangle \geq 0, \forall y \in C\} .
$$


In case $A=0$, problem (19) reduces to the equilibrium problem of finding $\hat{x} \in C$ such that

$$
f(\hat{x}, y) \geq 0, \quad \forall y \in C
$$

whose solution set is denoted by $\operatorname{EP}(f)$. When $f=0$, the generalized equilibrium problem becomes the variational problem of finding $\hat{x} \in C$ such that

$$
\langle A \hat{x}, y-\hat{x}\rangle \geq 0, \quad \forall y \in C,
$$

whose solution set is denoted by $\operatorname{VI}(C, A)$. For solving an equilibrium problem, we assume that the function $f$ satisfies the following conditions:

(A1) $f(x, x)=0, \forall x \in C$;

(A2) $f$ is monotone, that is, $f(x, y)+f(y, x) \leq 0, \forall x \in C$;

(A3) for all $x, y, z \in C, \lim \sup _{t \downarrow 0} f((1-t) x+t z, y) \leq f(x, y)$;

(A4) for all $x \in C, f(x, \cdot)$ is convex and lower semicontinuous.

The following Lemma 4.1 appears implicitly in Blum and Oettli [11] and is proved in detail by Aoyama et al. [12], while Lemma 4.2 is Lemma 2.12 of Combettes and Hirstoaga [13].

Lemma 4.1 [11,12] Let $f: C \times C \rightarrow \mathbb{R}$ be a function satisfying conditions (A1)-(A4), and let $r>0$ and $x \in \mathcal{H}$. Then there exists a unique $z \in C$ such that

$$
f(z, y)+\frac{1}{r}\langle y-z, z-x\rangle \geq 0, \quad \forall y \in C .
$$

Lemma 4.2 [13] Let $f: C \times C \rightarrow \mathbb{R}$ be a function satisfying conditions (A1)-(A4). For $r>0$, define $J_{r}^{f}: \mathcal{H} \rightarrow C$ by

$$
J_{r}^{f} x=\left\{z \in C: f(z, y)+\frac{1}{r}\langle y-z, z-x\rangle \geq 0, \forall y \in C\right\}
$$

for all $x \in \mathcal{H}$. Then the following hold:

(a) $f_{r}^{f}$ is single-valued;

(b) $f_{r}^{f}$ is firmly nonexpansive;

(c) $F\left(f_{r}^{f}\right)=\mathrm{EP}(f)$;

(d) $\operatorname{EP}(f)$ is closed and convex.

We call $f_{r}^{f}$ the resolvent of $f$ for $r>0$. Using Lemmas 4.1 and 4.2, Takahashi et al. [9] established the lemma below.

Lemma 4.3 [9] Let $f: C \times C \rightarrow \mathbb{R}$ be a function satisfying conditions (A1)-(A4) and define a set-valued mapping of $\mathcal{H}$ into itself by

$$
G_{f}(x)= \begin{cases}\{z \in \mathcal{H}: f(x, y) \geq\langle y-x, z\rangle, \forall y \in C\}, & \forall x \in C, \\ \varnothing, & \forall x \notin C .\end{cases}
$$

Then the following hold:

(a) $G_{f}$ is a maximal monotone operator with $\mathcal{D}\left(G_{f}\right) \subseteq C$;

(b) $\operatorname{EP}(f)=G_{f}^{-1} 0$;

(c) $J_{r}^{G_{f}} x=J_{r}^{f} x$ for all $x \in \mathcal{H}$. 
Theorem 4.4 Suppose that

(4.4.1) $f: C \times C \rightarrow \mathbb{R}$ is a function satisfying conditions (A1)-(A4) and $B: C \rightarrow 2^{\mathcal{H}}$ is a maximal monotone operator with $\mathcal{D}(B) \subseteq C$;

(4.4.2) $\mathrm{g}: \mathcal{H} \rightarrow \mathcal{H}$ is a $k$-contraction, $A: C \rightarrow \mathcal{H}$ is an $\alpha$-inverse strongly monotone mapping, and $V: \mathcal{H} \rightarrow \mathcal{H}$ is a $\bar{\gamma}$-strongly monotone and L-Lipschitzian mapping with $\bar{\gamma}>0$ and $L>0$;

(4.4.3) $T: C \rightarrow C$ is a $\lambda$-hybrid mapping;

(4.4.4) $\Omega:=F(T) \cap(A+B)^{-1} 0 \cap \operatorname{EP}(f) \neq \varnothing$;

(4.4.5) $\mu$ and $\gamma$ are two real numbers satisfying $0<\mu<\frac{2 \bar{\gamma}}{L^{2}}$ and $0<\gamma<\frac{\bar{\gamma}-\frac{L^{2} \mu}{2}}{k}$.

Start with any $x_{1} \in \mathcal{H}$ and define a sequence $\left\{x_{n}\right\}$ iteratively by

$$
\left\{\begin{array}{l}
f\left(u_{n}, y\right)+\frac{1}{r_{n}}\left\langle y-u_{n}, u_{n}-x_{n}\right\rangle \geq 0, \quad \forall y \in C, \\
z_{n}=J_{\sigma_{n}}^{B}\left(I-\sigma_{n} A\right) u_{n}, \quad n \in \mathbb{N}, \\
y_{n}=\frac{1}{n} \sum_{i=0}^{n-1} T^{i} z_{n}, \quad n \in \mathbb{N}, \\
x_{n+1}=\alpha_{n} \gamma g\left(x_{n}\right)+\left(I-\alpha_{n} V\right) y_{n},
\end{array}\right.
$$

where the sequences $\left\{\alpha_{n}\right\},\left\{\sigma_{n}\right\}$ and $\left\{r_{n}\right\}$ verify the following conditions:

(4.4.6) $\left\{\alpha_{n}\right\}$ is a sequence in $[0,1]$ with $\lim _{n \rightarrow \infty} \alpha_{n}=0$ and $\sum_{n=1}^{\infty} \alpha_{n}=\infty$;

(4.4.7) $\left\{\sigma_{n}\right\}$ and $\left\{r_{n}\right\}$ are sequences in $(0, \infty)$ so that $\liminf _{n \rightarrow \infty} r_{n}>0$ and there are two $a, b \in \mathbb{R}$ with $0<a \leq \sigma_{n} \leq b<2 \alpha$ for all $n \in \mathbb{N}$.

Then the sequence $\left\{x_{n}\right\}$ constructed by algorithm (20) converges strongly to a point $p_{0} \in \Omega$, where $p_{0}$ is the unique fixed point of $P_{\Omega}(I-V+\gamma g)$, and this point $p_{0}$ is also a unique solution of the hierarchical inequality

$$
\langle(V-\gamma g) p, q-p\rangle \geq 0, \quad \forall q \in \Omega
$$

Proof The set-valued mapping $G_{f}$ associated with $f$ defined in Lemma 4.3 is a maximal monotone operator with $\mathcal{D}\left(G_{f}\right) \subseteq C$, and it follows from Lemmas 4.2 and 4.3 that $F\left(J_{r}^{G_{f}}\right)=$ $F\left(J_{r}^{f}\right)=\operatorname{EP}(f)=G_{f}^{-1} 0$ for any $r>0$. Putting $G=G_{f}$ in Theorem 3.1, we see that $u_{n}=J_{r_{n}}^{G_{f}} x_{n}$, and so the conclusion follows from Theorem 3.1.

Since $C$ is a nonempty closed convex subset of $\mathcal{H}$, the indicator function $\iota_{C}$ defined by

$$
\iota_{C}(x)= \begin{cases}0, & x \in C \\ \infty, & x \notin C\end{cases}
$$

is a proper lower semicontinuous convex function, and its subdifferential $\partial \iota_{C}$ defined by

$$
\partial \iota_{C}(x)=\left\{z \in \mathcal{H}:\langle y-x, z\rangle \leq \iota_{C}(y)-\iota_{C}(x), \forall y \in \mathcal{H}\right\}
$$

is a maximal monotone operator, $c f$. Rockafellar [14]. As shown in Lin and Takahashi [3] the resolvent $J_{r}^{\partial \iota_{C}}$ of $\partial \iota_{C}$ for $r>0$ is the same as the metric projection $P_{C}$.

Theorem 4.5 Suppose that

(4.5.1) $f: C \times C \rightarrow \mathbb{R}$ is a function satisfying conditions (A1)-(A4); 
(4.5.2) $\mathrm{g}: \mathcal{H} \rightarrow \mathcal{H}$ is a $k$-contraction, $A: C \rightarrow \mathcal{H}$ is an $\alpha$-inverse strongly monotone mapping, and $V: \mathcal{H} \rightarrow \mathcal{H}$ is a $\bar{\gamma}$-strongly monotone and L-Lipschitzian mapping with $\bar{\gamma}>0$ and $L>0$;

(4.5.3) $T: C \rightarrow C$ is a $\lambda$-hybrid mapping;

(4.5.4) $\Omega:=F(T) \cap \operatorname{VI}(C, A) \neq \varnothing$;

(4.5.5) $\mu$ and $\gamma$ are two real numbers satisfying $0<\mu<\frac{2 \bar{\gamma}}{L^{2}}$ and $0<\gamma<\frac{\bar{\gamma}-\frac{L^{2} \mu}{2}}{k}$. Start with any $x_{1} \in \mathcal{H}$ and define a sequence $\left\{x_{n}\right\}$ iteratively by

$$
\left\{\begin{array}{l}
z_{n}=P_{C}\left(I-\sigma_{n} A\right) P_{C} x_{n}, \quad n \in \mathbb{N}, \\
y_{n}=\frac{1}{n} \sum_{i=0}^{n-1} T^{i} z_{n}, \quad n \in \mathbb{N}, \\
x_{n+1}=\alpha_{n} \gamma g\left(x_{n}\right)+\left(I-\alpha_{n} V\right) y_{n},
\end{array}\right.
$$

where the sequences $\left\{\alpha_{n}\right\},\left\{\sigma_{n}\right\}$ and $\left\{r_{n}\right\}$ verify the following conditions:

(4.5.6) $\left\{\alpha_{n}\right\}$ is a sequence in $[0,1]$ with $\lim _{n \rightarrow \infty} \alpha_{n}=0$ and $\sum_{n=1}^{\infty} \alpha_{n}=\infty$;

(4.5.7) $\left\{\sigma_{n}\right\}$ is a sequence in $(0, \infty)$ so that there are two $a, b \in \mathbb{R}$ with

$$
0<a \leq \sigma_{n} \leq b<2 \alpha \text { for all } n \in \mathbb{N} \text {. }
$$

Then the sequence $\left\{x_{n}\right\}$ constructed by algorithm (21) converges strongly to a point $p_{0} \in \Omega$, where $p_{0}$ is the unique fixed point of $P_{\Omega}(I-V+\gamma g)$, and this point $p_{0}$ is also a unique solution of the hierarchical inequality

$$
\langle(V-\gamma g) p, q-p\rangle \geq 0, \quad \forall q \in \Omega
$$

Proof Put $B=G=\partial \iota_{C}$ in Theorem 3.1. Then, for $\sigma_{n}>0$ and $r_{n}>0$, we have that $J_{\sigma_{n}}^{B}=J_{r_{n}}^{\partial \iota C}=$ $P_{C}$. Furthermore, as shown in Theorem 12 in Lin and Takahashi [3], we have

$$
\left(\partial \iota_{C}\right)^{-1} 0=C \quad \text { and } \quad\left(A+\partial \iota_{C}\right)^{-1} 0=\mathrm{VI}(C, A) .
$$

Thus we obtain the desired results from Theorem 3.1.

\section{Competing interests}

The author declares that they have no competing interests.

Received: 28 December 2013 Accepted: 1 July 2014 Published: 22 Jul 2014

\section{References}

1. Takahashi, W: Nonlinear Functional Analysis. Yokohama Publishers, Yokohama (2000)

2. Aoyama, K, lemoto, S, Kohsaka, F, Takahashi, W: Fixed point and ergodic theorems for $\boldsymbol{\lambda}$-hybrid mappings in Hilbert spaces. J. Nonlinear Convex Anal. 11, 335-343 (2010)

3. Lin, L, Takahashi, W: A general iterative method for hierarchical variational inequality problems in Hilbert spaces and applications. Positivity 16, 429-453 (2012)

4. Tian, M: A general iterative algorithm for nonexpansive mappings in Hilbert spaces. Nonlinear Anal. 73, 689-694 (2010)

5. Manaka, H, Takahashi, W: Weak convergence theorems for maximal monotone operators with nonspreading mappings in a Hilbert space. CUBO 13,11-24 (2011)

6. Liu, H, Wang, J, Feng, Q: Strong convergence theorems for maximal monotone operators with nonspreading mappings in a Hilbert space. Abstr. Appl. Anal. 2012, Article ID 917857 (2012). doi:10.1155/2012/917857

7. Goebel, K, Reich, S: Uniform Convexity, Hyperbolic Geometry, and Nonexpansive Mappings. Dekker, New York (1984)

8. Goebel, K, Kirk, WA: Topics in Metric Fixed Point Theory. Cambridge University Press, Cambridge (1990)

9. Takahashi, S, Takahashi, W, Toyoda, M: Strong convergence theorems for maximal monotone operators with nonlinear mappings in Hilbert spaces. J. Optim. Theory Appl. 147, 27-41 (2010)

10. Xu, HK: Iterative algorithms for nonlinear operators. J. Lond. Math. Soc. (2) 66, 240-256 (2002)

11. Blum, E, Oettli, W: From optimization and variational inequalities to equilibrium problems. Math. Stud. 63, 123-145 (1994) 
12. Aoyama, K, Kimura, T, Takahashi, W: Maximal monotone operators and maximal monotone functions for equilibrium problems. J. Convex Anal. 15, 395-409 (2008)

13. Combettes, PL, Hirstoaga, SA: Equilibrium programming in Hilbert spaces. J. Nonlinear Convex Anal. 6, 117-136 (2005)

14. Rockafellar, RT: On the maximal monotonicity of subdifferential mappings. Pac. J. Math. 33, 209-216 (1970)

10.1186/1029-242X-2014-264

Cite this article as: Hong: A general iterative algorithm for monotone operators with $\lambda$-hybrid mappings in Hilbert spaces. Journal of Inequalities and Applications 2014, 2014:264

Submit your manuscript to a SpringerOpen ${ }^{\odot}$ journal and benefit from:

- Convenient online submission

- Rigorous peer review

- Immediate publication on acceptance

- Open access: articles freely available online

- High visibility within the field

- Retaining the copyright to your article

Submit your next manuscript at $>$ springeropen.com 